Dickinson, David L. (1998) The Voluntary Contributions Mechanism with Uncertain Group Payoffs, Journal of Economic Behavior and Organization, 35(4): 517-33. (May 1998) Published by Elsevier (ISSN: 1879-1751).

\title{
The voluntary contributions mechanism with uncertain group payoffs
}

David L. Dickinson

\begin{abstract}
This paper reports the results of an experimental study which introduces an endogenous probability of public good provision into the voluntary contributions mechanism. Specifically, the two treatments allow for nonprovision even with positive contributions. In one treatment, the provision probability rises with increased contributions (while preserving the dominant strategy equilibrium of zero contributions). The results show that uncertainty per se lowers individual but not group contributions, lagged marginal incentives significantly predict contributions, and individuals significantly react to own-deviations from average group contributions. This has implications for work teams or professional sports teams who see increased probability of group rewards given higher effort levels.
\end{abstract}




\section{INTRODUCTION}

In standard public goods experiments, there is always uncertainty about the ultimate size of the group payoff. This group payoff is uncertain to the contributor in as much as he does not know how other group members will behave, but this is typically the only uncertainty that exists. Provision point (or threshold) experiments require a group to reach a certain total level of contributions before the public good is provided. Isaac et al. (1988), among others, consider provision points in an otherwise standard voluntary contributions mechanism (VCM) experimental design. Even though individual incentives do not support complete contribution towards the public good as a noncooperative outcome, increases in thresholds typically increase contribution levels and decrease the probability of reaching the threshold. Once the group contribution is determined the probability of provision is either 0 or 1 . In threshold experiments it is also possible to experience nonprovision of the public good with positive contribution levels, but such experiments also fundamentally change the equilibrium environment of the VCM. Typically, multiple equilibria will exist, none of which is dominant.[1]

This paper reports the results of an experimental study in which a different type of uncertainty is introduced into the VCM environment - uncertainty in the provision of the public good conditional on aggregate contributions. In one treatment we are able to examine how subjects behave when the ex post probability of provision is between 0 and 1 . The hypothesis of riskaverse subjects leads to a prediction of lower contributions in this treatment.[2] The second treatment includes a variable probability of provision that increases in contribution levels. An interesting byproduct of this treatment is that the marginal incentive to contribute to the public good (the marginal per capita return of Isaac et al., 1984) also increases in contribution levels. If subjects base current decisions on past information, then we predict that high (low) contribution levels will cause continued high (low) contribution levels given these incentives. Environments with this uncertainty in public goods provision are motivated by considering cooperative problems such as the following:[3]

1. A high-paid professional athlete could give up part of his salary so that owners could fill a more balanced roster with a higher probability of winning the ultimate group payoff, a championship.

2. A work team formed at an auto plant has individual compensation tied to group results. Individuals contribute their effort towards achieving a group incentive bonus, but the correlation between effort and results is imperfect.

3. A conservation group spends time and effort attempting to save a particular animal from extinction. With their efforts the probability of extinction of the species is lowered and at least some groups of people are better off as a result.

These are all cases in which people give up private payoffs for the chance of receiving a higher group payoff. Additionally, the more effort contributed towards achieving the public good, the higher the probability of success - in some cases even, the higher the probability of receiving an even larger public good (as with sports teams).[4] A possible implication of such uncertainty is that contributions or effort would decline if individuals are risk-averse (see Section 2 ). Also, in a 
repeated setting that parallels the repeated nature of many team work projects, individuals may learn from previous rounds. Such learning may be shaped by group contribution levels, owncontributions relative to group contribution levels, and/or psychological factors that may alter expected payoffs (e.g. such as nonprovision of the public good).

Upon analyzing the results of ten experimental sessions we find that the introduction of a static ex post probability of provision of less than one significantly decreases contributions at the individual level (insignificantly at the group level). This suggests risk aversion. Instances of nonprovision do not significantly alter contribution levels in the subsequent round of decision making. There is also a strong marginal incentive effect suggesting that previous contribution levels may be used to make current contribution decisions. As in existing studies of the VCM without communication, there is decay of contributions upon repetition.[5] Given that the uncertainty in the provision of the public good may lower contributions, work teams may face lower contributions of effort than would otherwise be the case.

One additional factor shown to affect individual contributions is the deviation of a person's contributions in the previous round from the residual group average. This, along with the marginal incentive effect, is appealing intuitively since it suggests that people use feedback from the other members of their group as a means, at least to some extent, of shaping their future contribution levels.

\section{EXPERIMENTAL DESIGN}

In this particular design, groups of 5 subjects are given a per period endowment of 15 tokens which can be placed in a private account (earning 1 cent for that subject alone) or a group account (generating 1/2 cent for each group member). Thus, the group account represents the public good. In the event that the group payoff occurs with probability $p$, the general form of the payoff function that each individual faces in an experimental period (round) is,

$$
U_{i}=q\left(w_{i}-m_{i}\right)+p\left(\frac{a \sum_{i} m_{i}}{N}\right)
$$

where $w_{i}$ is the initial endowment of tokens per period, $m_{i}$ is the per period message or contribution to the group account (and therefore, $\sum m_{i}$ is the size of the public good), $q$ is a conversion parameter for the private account payoffs, $a$ is a conversion parameter for the group account payoff, and $p$ is the probability of provision. In the parameterization described above

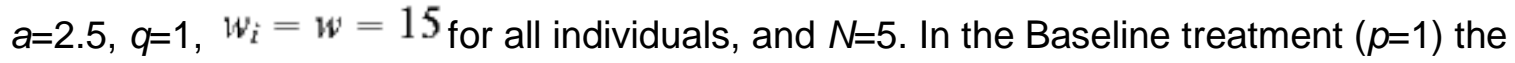
Marginal Per Capita Return $(M), M=\left(a / q_{n}\right)=1 / 2$, which is just the $1 / 2$ cent that is generated for each member of the group when one individual contributes a token.[6] It can also be seen as the marginal rate of substitution of the private good for the public good. This 
measure $M$ is introduced by Isaac et al., where they refer to it as the MPCR. In this design, the single-period dominant strategy is to contribute nothing, while the group optimum payoff is achieved when contributions are 100 percent. The group payoff in this case would be $\$ 0.375$ for each group member for each period while the free-riding Nash equilibrium would yield each subject $\$ 0.15$ per period.

For subsequent treatments we now define $E M$ as the expected marginal per capita return

$$
E M=E(M)=E\left(-\frac{\partial U / \partial y}{\partial U / \partial m_{i}}\right)=\frac{p a}{q N} \text { where } y=\sum_{i} m_{i}=
$$

contributions to the public good.

This is just the expected value of the marginal rate of substitution between the private and the public good. In the Baseline design where $p=1, E M=M$. The introductory examples used to motivate the inclusion of uncertainty of public good provision into the VCM reflect cases in which the probability of provision should increase in contribution levels. However, since such a treatment actually alters two experimental parameters, we first discuss a treatment that allows for an uncertain ex post probability of provision, but does not allow for a variable probability of public goods provision.

\section{The Uncertainty Treatment}

Let the treatment where $p<1$ and is fixed, be called the Uncertainty treatment. For this Uncertainty treatment in the experiment that follows $p=0.70$. By altering the parameter a the $E M$ is held to $1 / 2$, thereby preserving the single-period dominant strategy of zero contributions and the Pareto optimal outcome at 100 percent contributions. This treatment isolates the effect of altering the probability of a group payoff.

While this treatment was originally conceived to bridge the gap between the Baseline and the treatment to follow, it is interesting in and of itself as it provides a clearcut prediction of subject behavior. Specifically, comparing the Uncertainty treatment with the Baseline presents the subjects with a mean preserving spread on the distribution of possible Marginal Per Capita Returns. Therefore, risk-averse subjects are predicted to contribute less in the Uncertainty treatment. It is shown in A that any subject with a concave utility function (not restricted to just quadratic utility functions) would prefer the distribution of Marginal Per Capita Returns, and hence is predicted to contribute more, under the Baseline treatment than under the Uncertainty treatment.[7] This may seem counterintuitive given that the Nash prediction is that subjects should contribute zero in both the Baseline and the Uncertainty treatment. However, Isaac et al. among others, document the importance of their MPCR in predicting contributions in VCM environments with a full free-riding equilibrium prediction. Given this, it would be prudent to examine any positive contributions in the light of the aforementioned risk-averse prediction. 


\section{The Incentive Treatment}

In the final experimental treatment there is not only uncertainty in the provision of the group payoff, but the probability of provision also increases in the level of group contributions. Let us call this the Incentive treatment. The probability $p$, as a function of total contributions, is chosen as

$$
p=\frac{\alpha \sum_{i} m_{i}}{N w}
$$

This choice of $p$ has the property that $p \in[0, \alpha]$ and $p$ increases in the size of the public good. A possibility would be to choose $\alpha=1$. However, the motivation earlier suggests that the probability of provision should never reach one, even with complete contributions. Therefore, let $\alpha=0.75$. Given that there are 75 total tokens distributed to the group members, $\alpha=0.75$ ensures that each additional token contributed adds exactly 1 percent to the value of $p$ and so $p \in[0,75]$. Note that $p$ strictly increases in the size of the public good since $\left(\partial p / \partial m_{i}\right)=(\alpha / N w)$ when $\alpha>0$ so that contributing not only increases the expected value of the group payoff but higher contributions give the subjects a higher probability of sharing a larger pie. The expected period payoff to the subject is

$$
U_{i}=q\left(w-m_{i}\right)+\left(\frac{\alpha \sum_{i} m_{i}}{N w}\right)\left(\frac{a \sum_{i} m_{i}}{N}\right)
$$

The parameter $a$ is altered for this treatment so that $a=3.33$. Obviously we cannot hold $E M$ constant at 0.50 since $E M$ is now a function of a probability that varies with the number of total contributions to the group account, but this parameterization yields $E M=0.50$ when exactly half of all tokens (37.5) are contributed towards the group account. For lower levels of contributions, $E M<0.50$ and for higher levels $E M>0.50$. The parameter values for this treatment are chosen such that $E M \in[0,1]$ and we preserve the dominant strategy to contribute nothing.[8]

The socially optimal choice is still for all subjects to contribute all of their tokens to the group account (i.e. it would maximize the expected value of total group earnings).[9] We see this by noting that the new social problem is

$$
\underset{\sum_{i} m_{i}}{\operatorname{Max}} E\left(\sum_{i} U_{i}\right)=\underset{\sum_{i} m_{i}}{\operatorname{Max}} q\left(N w-\sum_{i} m_{i}\right)+\left(\frac{\alpha \sum_{i} m_{i}}{N w}\right) a \sum_{i} m_{i}
$$


This is obviously maximized by choosing $\sum_{i} m_{i}=75$ (i.e. total contribution by all subjects).

The expected value of the sum of individual utilities is now a convex function which achieves its maximum at the upper boundary. This function is shown graphically in Fig. 1 along with the social maximization problem of the Baseline and Uncertainty treatments. More intuitively, we see from Eq. 5 that at any level of contributions, contributing one more token yields $p \cdot a-q$ in extra expected group payoffs. Given that $q=1$ and $a=3.33$, as long as $p>0.3$ (and it will increase with more contributions) the expected value of total payoffs will be greater than the full free-

riding level. The probability of provision is greater than or equal to 0.3 with $\sum_{i} m_{i} \geq 30$, and therefore the highest expected total payoff is when everything is contributed and the expected total payoffs are 187.5 cents per period (versus total payoffs of 75 cents per period when subjects contribute nothing.

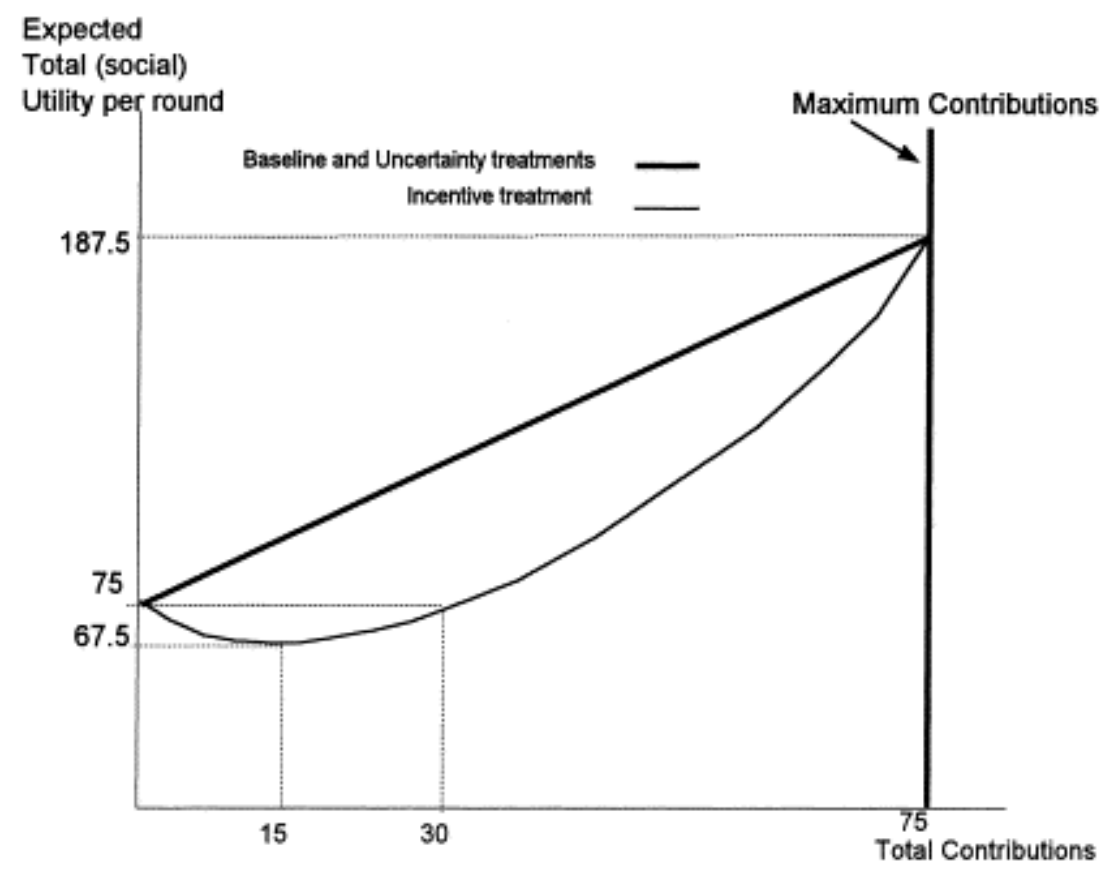

Fig. 1. Behavior of expected social utility

The shape of the group payoff function in the Incentive treatment provides an alternative explanation as to why subjects may not contribute towards the group account. Given that there is both a global and local maximum, subjects in low contributing groups actually increase the total group payoff by free-riding if they do not believe that the group is capable of surpassing this threshold level of contributions. Any possible marginal incentive effects should not be ignored, however, since low contributions would tend to promote low contributions in future rounds given that they have the effect of lowering the EM. In other words, learning from previous rounds may be an important determinant of contributions. 


\section{EXPERIMENTAL PROCEDURES}

Ten experiments were conducted using undergraduate students at the University of Arizona. The experiments were handrun and the subjects were inexperienced in the institution. Sessions (including instructions) lasted approximately one hour and the average payoff of all sessions was $\$ 14.20$, ranging from a low of $\$ 10$ to a high of $\$ 21$. Groups of 5 subjects were given Instructions, a Decision and Earnings Sheet, and a Returns from the Group Account Sheet.[10] The parameters for the Baseline, the Uncertainty, and the Incentive treatments are as stated in Section 2 of the paper. The logistics of their implementation are discussed here.

In the Baseline, once subjects make their decision in each period, the experimenter goes around the room and totals all of the contributions of tokens towards the Group Account. This total is then written on the blackboard at the front of the room. Given this total, subjects can then refer to the appropriate entry in a 'Returns from the Group Account' table to find out how much they earn that period from the Group Account. Subjects also know what Private Account tokens are worth and so that they can then find their total earnings for that period. This procedure is repeated for 7 periods.

After the initial Baseline periods (done both to replicate previous results and to provide a benchmark for comparisons) subjects are handed a new instructions, a new Returns from the Group Account table, and a new Decisions and Earnings Sheet. In the Uncertainty treatment, everything is the same as in the Baseline except for the probability draw, which is done manually with a bingo cage. The subjects' attention is drawn to a bingo cage at the front of the room prior to any decisions being made. Subjects are asked to witness the placement of 10 balls numbered 1-10 into the cage. Now, after each period's total tokens contributed towards the Group Account have been written on the blackboard, a draw is made from the bingo cage. If the number 8, 9, or 10 is drawn, subjects earn nothing from the Group Account for that period. Subjects witness this draw and the subsequent replacement of the ball into the cage. This procedure is repeated for 7 periods.

In the Incentive treatment, all procedures are the same as in the Uncertainty treatment with a slight variance in the probability draw. Prior to the beginning of this portion of the experiment, subjects witness the placing of balls numbered 1-100 into a bingo cage at the front of the room. Upon the experimenter writing the total number of tokens contributed towards the Group Account on the blackboard, the probability of provision is calculated as the total number of tokens contributed towards the Group Account for that period divided by 100. In order for the Group Account to generate any earnings for the subjects, the draw must be less than or equal to the total number of tokens contributed. For example, if the probability of provision is determined to be 0.27 , then the Group Account would generate earnings if and only if a ball numbered 1-27 were drawn from the bingo cage. If a ball numbered 28-100 were drawn, then the Group Account would generate no earnings for any subject for that period. Subjects witness the draw from the bingo cage and the subsequent replacement of the ball. This procedure is repeated for 7 periods.

The Baseline is what the subjects first experience, but the ordering of the final two treatments is altered for different groups of subjects. This is a simple control for ordering effects in the 
probability treatments. The possibility of an ordering effect does exist since the Baseline is always the first set of rounds, but familiarizing the subjects with the basic environment was considered important before introducing the probability treatments (furthermore, ordering is not apparent at all in the data). At the end of the final period of the final treatment, subjects' earnings are totaled and the subjects are paid in cash.

\section{EXPERIMENTAL RESULTS}

The data aggregated across all ten experiments are shown graphically in Fig. 2. We can see from Fig. 2 that initial contribution levels are significantly greater than zero, and there is a decay in contribution levels when approaching the final rounds.

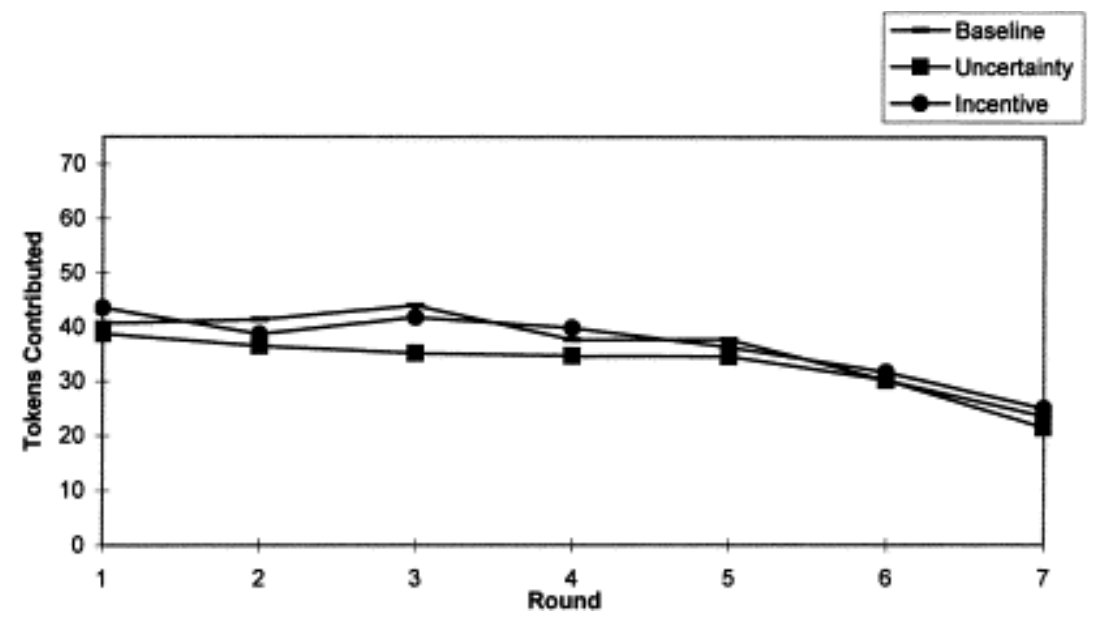

Fig. 2. Aggregate contribution Levels by treatment.

A Wilcoxon signed-rank test rejects the null hypothesis of equal mean contribution levels between the Uncertainty and Incentive treatments $(p=0.10)$ in favor of the alternative that contributions are lower in the Uncertainty treatment. We fail to reject the null hypothesis of equal mean contribution levels when comparing the two treatments with the Baseline. The conclusions of this nonparametric test do not support the risk-averse hypothesis that subjects will contribute less in the Uncertainty treatment than in the Baseline.

The first 4 rounds of each treatment from Fig. 2 appear to suggest that there are lower contributions in at least the first few rounds of the Uncertainty treatment. A similar signed ranktest only using data from the first 4 rounds confirms this by finding that contributions are significantly lower in the Uncertainty treatment (compared to the Baseline, $p=0.10$ ). In the last 3 rounds, we fail to reject the null hypothesis that mean contributions are the same in the Baseline and Uncertainty treatment $(p=0.40)$. In fact, we can see in Fig. 2 that contribution levels are quite similar across all treatments in the final 3 rounds. Table 1 shows the mean level of contributions over the last 3 rounds of each session for all experiments and treatments. 
Table 1. Mean contribution levels for the final 3 rounds of each session (by treatment)

\begin{tabular}{lccc}
\hline Experiment \# & Baseline & Uncertainty & Incentive \\
\hline 1 & 40.7 & 33.7 & 45.0 \\
2 & 31.3 & 46.3 & 43.0 \\
3 & 37.7 & 22.0 & 62.3 \\
4 & 35.7 & 43.3 & 53.7 \\
5 & 13.3 & 9.7 & 4.0 \\
6 & 29.3 & 39.0 & 39.3 \\
7 & 19.3 & 14.7 & 1.3 \\
8 & 22.3 & 33.3 & 14.3 \\
9 & 23.7 & 14.0 & 6.7 \\
10 & 51.0 & 24.3 & 39.7 \\
Overall Average & 30.4 & 28.0 & 30.9 \\
\hline
\end{tabular}

\section{Group Contributions Modeling}

In analyzing the group-level data, a fixed effects model is used in which each different experimental group is allowed a fixed effect.[11] Tokens contributed by the entire group are assumed to be a simple linear function of the round-number inside the treatment in which they occur, the experiment, the treatment, the one-period lagged EM, and whether or not the public good was provided in the previous round. The ex ante logic of this last variable is that nonprovision, even with positive contributions, may psychologically affect individuals even though we may not predict that it would economically affect them. The base equation of estimation is

(6)

$$
\begin{aligned}
& \text { tokens } \left._{i j t}=\alpha_{j}+\beta_{1} \text { (round dummies }\right)+\beta_{2} \text { Nopublic }+ \\
& \beta_{3} \text { Uncert }+\beta_{4} \text { Incent }+\beta_{5} E M_{i j, t-1}+\varepsilon_{i j t}
\end{aligned}
$$

$$
\begin{array}{cl}
\text { where } i & =\text { treatment (Baseline, Uncertainty, or Incentive) } \\
j & =\text { group (experiment number) } \\
t & =\text { round number }(2-7)
\end{array}
$$

The Round Dummies consist of dummy variables for rounds $3,4,5,6$, and 7 . Therefore, $\beta_{1}$ is actually a vector of 5 coefficients (simplified in Eq. 6 for exposition) - one for each round dummy variable. The Round Dummies allow for the possibility of each round having a different effect on the decay of contributions. The variable Nopublic is a dummy variable with value 1 if the public good was not provided in the previous round. Uncert and Incent are dummy variables for the Uncertainty and Incentive treatments, respectively. The variable EM is the one-period lagged 
value of EM. While this is constant at 0.5 for both the Baseline and the Uncertainty treatments, it is variable for the Incentive treatment. As such, we are implicitly assuming that expectations of current-round group contributions are based on group contributions of the previous round. Isaac et al. (1984), Kim and Walker (1984), among others, study the importance of marginal incentives on contributing behavior. A higher marginal incentive to contribute, even while preserving the dominant strategy Nash prediction of zero contributions, significantly increases contributions. This leads us to hypothesize that the coefficient on Incent will be positive if subjects use past history in contributing decisions. The error term ${ }^{\varepsilon_{i j t}}$ is assumed to be a normal i.i.d. error term with the usual properties.

The results of the estimation are presented in Table 2. OLS estimation of equation Eq. 6 revealed first-order autocorrelation (across rounds, holding $i$ and $j$ constant), so the PraiseWinsten transformation is used to perform the full GLS estimation. The number of useable observations for analysis is 180.[12]

Table 2. Results from fixed effects model estimation of group data

\begin{tabular}{lclc}
\hline Variable & Coeff. $(p$-value) & Fixed effects & Coeff. $(p$-value) \\
\hline Round3 & $2.70(0.18)$ & Experiment 1 & $29.44(0.00)^{\mathrm{a}}$ \\
Round4 & $-0.59(0.80)$ & Experiment 2 & $27.33(0.00)^{\mathrm{a}}$ \\
Round5 & $-1.47(0.53)$ & Experiment 3 & $25.72(0.00)^{\mathrm{a}}$ \\
Round6 & $-6.26(0.01)^{\mathrm{a}}$ & Experiment 4 & $32.50(0.00)^{\mathrm{a}}$ \\
Round7 & $-12.88(0.00)^{\mathrm{a}}$ & Experiment 5 & $1.81(0.35)^{\mathrm{a}}$ \\
Nopublic & $-0.03(0.98)$ & Experiment 6 & $25.85(0.00)^{\mathrm{a}}$ \\
Uncert & $-2.75(0.15)$ & Experiment 7 & $7.10(0.05)^{\mathrm{a}}$ \\
Incent & $0.01(0.99)$ & Experiment 8 & $16.30(0.00)^{\mathrm{a}}$ \\
EM & $41.04(0.00)^{\mathrm{a}}$ & Experiment 9 & $7.80(0.05)^{\mathrm{a}}$ \\
$\rho$ & 32 & Experiment 10 & $22.02(0.00)^{\mathrm{a}}$ \\
Adjusted $R^{2}$ & 0.71 & &
\end{tabular}

Number of observations $=180$

Actual provision percentage of all rounds in Uncertainty treatment $=0.70$

Actual provision percentage of all rounds in Incentive treatment $=0.41$

andicates statistical significance at least at the 0.05 level.

We see from Table 2 that the significant variables in this specification are many of the fixed effects terms, Round6, Round7, and EM. The fixed effects reflect the highly nonzero level of initial contributions present in all VCM experiments and are an important determinant, in magnitude, of contributions. The fixed effects are highly significant across groups $(p=0.00)$. The mean level fixed effect across experiments is 19.6 tokens contributed per group. The coefficients on Round 6 and Round 7 indicate significant decay in contributions with a decrease of 12.9 tokens per group in the final round.[13]

At the group level, we fail to reject the null hypothesis that mean contributions are the same under each treatment $(p=0.21)$. The insignificant positive coefficient on Uncert may seem surprising given our risk-averse hypothesis, but recall that nonparametric tests only found a 
significant treatment effect in the first 4 rounds. Nonprovision of the public good does not seem to statistically affect contributions either. The coefficient on Nopublic is negative but highly insignificant.[14] The fact that the treatment variables and the uncertainty of the public good's provision do not seem to affect contributions will be revisited in the next section where the data are modeled at the individual level. Such lack of significance would have important implications for work teams and group projects that might otherwise have reason for concern if there is inherent uncertainty in group payoffs.

While insignificant as a treatment variable, the Incentive treatment is the only treatment in which the variable EM is not constant. The coefficient on EM is significant and substantial in magnitude, suggesting that individuals do use past history in shaping current round contributions. This becomes especially important in the Incentive treatment as changes in the $E M$ have a significant effect on contribution levels. The coefficient's magnitude of about 41 implies that each increase in the EM of 0.10 would increase group contributions by about 4 tokens in the Incentive treatment. As such, low (high) contributions will promote more low (high) contributions which are consistent with the higher variance in contribution levels in the Incentive treatment (see Table 1).

In the study on marginal incentives by Isaac et al. (IWT) the average contribution level across 10 rounds for an experiment in which their MPCR $=0.3$ is 26 percent of total tokens, while when $\mathrm{MPCR}=0.75$ this increased to 58 percent of total tokens. This represents an increase in contributions of 32 percent. A similar increase in the $E M$ of 0.45 in the Incentive treatment is predicted to increase group contributions by 25 percent (i.e. by 18.5 out of a possible 75 tokens).[15] The changing EM within a treatment has not only the same directional effect on contributions, but an effect similar in magnitude to IWT.

\section{Individual Contributions Modeling}

The base equation of estimation for the individual data is

(7)

$$
\begin{aligned}
& \text { tokens }_{i k t}=\alpha_{k}+\beta_{1}(\text { Round Dummies })+\beta_{2} \text { Nopublic }+ \\
& \beta_{3} \text { Uncert }+\beta_{4} \text { Incent }+\beta_{5} E M_{i k, t-1}+\beta_{6} \text { Deviate }+\varepsilon_{i k t}
\end{aligned}
$$

$$
\begin{array}{cl}
\text { where } i & =\text { treatment (Baseline, Uncertainty, or Incentive) } \\
k & =\text { individual (50 individuals) } \\
t & =\text { round number }(2-7)
\end{array}
$$

The indices $i, k$, and $t$ are for the treatment (Baseline, Uncertainty, or Incentive), the individual (50 individuals), and the round number (2-7), respectively. Here, $\alpha_{\kappa}$ is now the individual specific fixed effect. 
In addition to the variables used in analysis of the group data, we include a variable that attempts to capture an individual's response to the signal being received from the other group members. Once individuals make their decisions and the total number of tokens contributed by the group is written on the black board, an individual can easily figure out how much they contributed relative to what others contributed. Given this, the variable Deviate is included to capture the individual's response to such a signal. Deviate is the deviation of an individual's contributions in the previous round from the average of the remaining group members in that round.[16] Thus, if an individual contributed more (less) than the average of the remaining 4 group members, Deviate will be positive (negative). The coefficient on Deviate is hypothesized to be negative if individuals are concerned with contributing what everyone else is contributing on average. With the individual data, the number of useable observations is 900.[17]

The results of the fixed effects model estimation of Eq. 7 are presented in Table 3.[18] Many of the coefficients on the individual fixed effects are significant and all of them are available from the author upon request. Table 3 shows again that significant decay in contributions occurs in the final rounds.

Table 3. Results from fixed effects model estimation of individual data

\begin{tabular}{lc}
\hline Variable & Coeff. ( $p$-value) \\
\hline Round3 & $0.38(0.34)$ \\
Round4 & $-0.39(0.33)$ \\
Round5 & $-0.45(0.26)$ \\
Round6 & $-1.43(0.00)^{\mathrm{a}}$ \\
Round7 & $-2.70(0.00)^{\mathrm{a}}$ \\
Nopublic & $0.03(0.92)$ \\
Uncert & $-0.71(0.02)^{\mathrm{a}}$ \\
Incent & $-0.14(0.66)$ \\
EM & $9.04(0.00)^{\mathrm{a}}$ \\
Deviate & $0.26(0.00)^{\mathrm{a}}$ \\
Adjusted $R^{2}$ & 0.55 \\
$N$ & 900 \\
\hline andicates significance at least at the \\
0.05 level.
\end{tabular}

The null hypothesis that mean contributions are the same under each treatment is rejected $(p=0.03)$. At the individual level, the coefficient on Uncert is negative and significant. Its magnitude suggests that contributions decline by about 2.5 tokens per group in the Uncertainty treatment. Both Incent and Nopublic insignificantly altered contributions. Individual fixed effects are significant across individuals as well $(p=0.00)$. The average level of all fixed effects is 3.34 tokens contributed per person.

Similar to the group-level model in Eq. 6, there is a strong marginal incentive effect reflected in the coefficient of 9.04 . Since this coefficient reflects a per person change in contributions, the 
size of the marginal incentive effect is similar in magnitude to that found in the estimation of Eq. 6. Also, the variable Deviate is highly significant and positive. It is small in magnitude, explaining $1 / 4$ of a token per individual, but the mere fact that it is positive offers some interesting insights into individual behavior in group settings. Given the general pattern of all subjects to show a decay in contributions across rounds of the experimental treatment, the coefficient on Deviate will be positive if above-average contributors have contributions that decline at a below-average rate while below-average contributors have contributions that decline at an above-average rate.[19] This would result if, for example, some individuals learn to free-ride (play the dominant strategy) more quickly than others. Deviations from the group mean will therefore not correct themselves, a fact that may lead one towards considering such tax and subsidy schemes as that found in Falklinger et al. (1995). However, differing abilities to contribute (i.e. different endowment levels) may render such schemes difficult to implement.[20]

Unfortunately, it is difficult to come to a final conclusion as to whether or not the Uncertainty treatment lowers contributions. This variable was sensitive to model specification at both the individual and group level. In an earlier model that replaced the variables $E M$ and Incent with an interaction variable ( $E M^{\star}$ Incent), the coefficient on Uncert was not statistically significant while the coefficient on Nopublic was statistically significant (and still negative). The slightly more behavioral specification of Eq. 7 is estimated as having a significant uncertainty effect. An uncertainty effect may exist, but this initial analysis does not offer it as a strong effect on contributions. Variables that were significant and robust with respect to specification include the dummy variables for rounds 6 and 7, EM, and Deviate.

\section{SUMMARY}

This experimental study examines the voluntary contribution mechanism for providing a special type of public good - one that is not provided with certainty even when individuals contribute much towards its provision. Such an environment mimics several real-world public goods environments that, until now, have escaped study.

The hypothesis that uncertainty, per se, will decrease contributions has not been fully answered in this paper. The hypothesis is supported at the individual level, but not at the group level, and it is sensitive to model specification. At best, it has a weak effect on contributions. The Incentive treatment, per se, does not significantly affect contribution levels either (although the changing marginal incentives that exist only in this treatment do). In this treatment, there is both a global social optimum at complete contributions and a local optimum at zero contributions. This feature of the institution, along with the lower expectation reflected by the lower $E M$, may further drive some groups to low contributions and thus explain the large variance in contributions across experiments in the Incentive Treatment.

Other than the significant decay in contributions towards the end rounds, the marginal incentive effects appear quite strong and robust. As a lagged variable, its significance sheds some light on how subjects use previous history in contribution decisions. High (low) contributions will promote further high (low) contributions. In addition, the positive (and significant) sign on 
Deviate implies that individuals do not merely converge towards a common group mean. Rather, contributions for different individuals decay at different rates. It is possible that some individuals 'learn' the dominant strategy quicker than others. Together these suggest that some type of learning through repetition may be involved that merits further attention in future research. In general, these results substantiate the importance of marginal incentives in environments which have a dominant strategy prediction of zero contributions.

These results also have implications on contribution levels in work teams. While perhaps only a weak effect, if uncertainty does lower contribution levels of work teams, work team managers might then contemplate alternative forms of compensation that to not involve the inherent uncertainty that exists when individual pay is linked to group outcomes and these same outcomes are imperfectly correlated with effort. Even more important are the implications of the marginal incentive effects found in this study. These effects show that low contributions promote more low contributions in the future. Any way of increasing contribution levels in these work team environments with uncertain group payoffs (e.g. by alternative forms of compensation or team organization) would prove beneficial since the rising marginal incentives would help sustain effort levels to the benefit of both manager and team member.

\section{ACKNOWLEDGMENTS}

I would like to especially thank the Russell Sage Foundation for funding the experiments in this paper. I would also like to thank Mark Isaac, Leslie Stratton, Price Fishback, two anonymous referees, and the participants in the Applied Economics Workshop at the University of Arizona for many valuable comments which have certainly improved the quality of this paper. All errors remain my own.

\section{REFERENCES}

Austen-Smith, D., 1980. , Individual contributions to public goods . Economic Letters 5, pp. 359361

Brown-Kruse, J., Hummels, D., 1992. Gender effects in public goods contribution: Do individuals put their money where their mouth is? Technical report, University of Colorado, Boulder, CO. Forthcoming, Journal of Economic Behavior and Organization

Chan, K., Mestelman, S., Moir, R., Muller, A., 1996. The voluntary provision of public goods under varying income distributions. Canadian Journal of Economics, 54-69.

Eichberger, J., Kelsey, D., 1996. Free riders do not like uncertainty. Working paper, The University of Birmingham

Falklinger, J., 1994. , The private provision of public goods when the relative size of contributions matters. Finanz Archiv 51 3, pp. 358-371 
Falklinger, J., Fehr, E., Gachter, S., Winter-Ebmer, R., 1995. A simple mechanism for the efficient private provision of public goods - Experimental evidence. Arbeitspapier NR. 9517.

Hadar, J. and Russell, W., 1971. , Stochastic dominance and diversification. Journal of Economic Theory 3, pp. 288-305

Isaac, R., Schmidtz, D. and Walker, J., 1988. , The assurance problem in a laboratory market. Public Choice 62 3, pp. 217-236

Isaac, M., Walker, J. and Thomas, S., 1984. , Divergent evidence on free riding: An experimental examination of possible explanations. Public Choice 43 1, pp. 113-149

Kim, O. and Walker, M., 1984. , The free rider problem: Experimental evidence. Public Choice 43, pp. 3-24

Ledyard, J., 1995. Public goods: A survey of experimental research. In: Kagel, J., Roth, A. (Eds.), The Handbook of Experimental Economics, Princeton University Press, Princeton, NJ, pp. 111-194

Ostrom, E., Walker, J. and Gardner, R., 1992. , Covenants with and without a sword: Selfgovernance is possible. American Political Science Review 86 2, pp. 404-417

Rothschild, M. and Stiglitz, J., 1970. , Increasing Risk: I. A Definition. Journal of Economic Theory 2, pp. 225-243

Saijo, T., Tatamitani, Y., 1991. Characterizing neutrality in the voluntary contribution mechanism, Working Paper, Institute of Socio-Economic Planning, University of Tsukuba, Tsukuba, Japan

Walker, J. and Gardner, R., 1992. , Probabilistic destruction of common pool resources:

Experimental evidence. The Economic Journal 102 414, pp. 1149-1161 


\section{APPENDIX A: STOCHASTIC DOMINANCE PREFERENCE AGAINST THE UNCERTAINTY TREATMENT}

This appendix shows that with the Baseline and Uncertainty treatments of the proposed design, a concept of stochastic dominance can give theoretical predictions of subject behavior under only mildly restrictive assumptions on subject preferences. Definitions of the concepts of FSD and SSD can be found in Hadar and Russell (1971) and additional discussions of risk and mean preserving spreads are in Rothschild and Stiglitz (1970). If $G(x)$ and $F(x)$ are two cumulative distribution functions (CDFs) of the continuous random variable $x$ defined on the range $R$ which represents a closed interval $[b, c], b<c$, then the probability density function $f(x)$ is said to be at least as large as $g(x)$ in the sense of SSD if

$$
\int_{b}^{x} F(t) \mathrm{d} t \leq \int_{b}^{x} G(t) \mathrm{d} t, \text { for all } x \in R
$$

The function $f(x)$ is said to be larger than $g(x)$ in the sense of SSD iff the above inequality holds strictly for at least one $x \in R$. The intuitive flavor is that $F(x)$ lies mostly below $G(x)$ for $f(x)$ to be preferred to $g(x)$.

In our particular application, $x$ is the discrete random variable representing the Marginal Per Capita Return (MPCR). The CDF represents the cumulative probability that the MPCR is less than or equal to a certain level $X$. Therefore, in the Baseline $x$ is the discrete random variable that takes on the value $(a / q n)$ with certainty under the distribution described by $F(x)$ (the Baseline CDF). In the Uncertainty Treatment (Section 2.1) $x$ takes on the values 0 or $(\hat{a} / q n)$ with probabilities $(1-p)$ and $p$, respectively, under the distribution $G(x)$ (the Uncertainty CDF). These discrete CDFs are shown in Fig. 3.

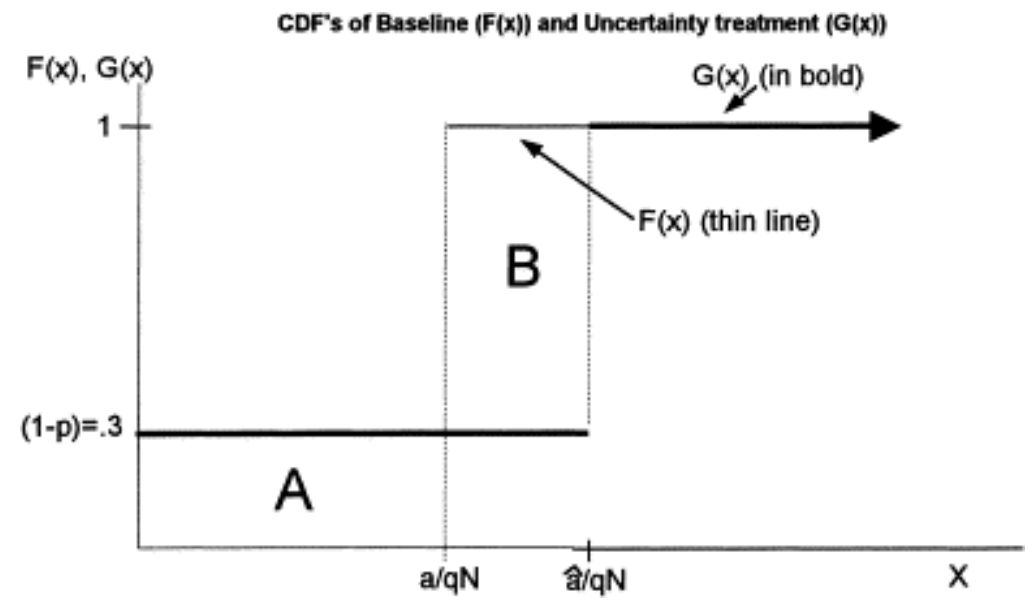

Fig. 3. CDF's of the expected marginal per capita return. 
The concept of FSD is obviously not applicable here since it requires one CDF to lie everywhere below another for a given class of preferences to prefer $f(x)$ to $g(x)$. However, we show that Eq. A.1 is satisfied in its discrete version.

Using Fig. 3, it is apparent that Eq. A.1 is equivalent to asking whether the area $B$ is less or equal to the area A. Subjects will then prefer (in the sense of SSD) the distribution of MPCRs under the Baseline to the distribution in the Uncertainty treatment if

(A.2)

$p\left(\frac{\hat{a}-a}{q N}\right) \leq(1-p) \frac{a}{q N}$

or

$p \hat{a} \leq a$

This holds for all values of $x$ up to $(\hat{a} / q N)$ (with the aforementioned parametrization), which is the upper bound of the cumulative distribution function $G(x)$. At $x=(\hat{a} / q N)$ Eq. A.2 holds as an equality (since this is the upper limit of $G(x)$ and both CDFs must integrate to 1 ). This shows that for the two functions $f(x)$ and $g(x)$ Eq. A.1 is satisfied and therefore $f(x)$ is preferred to $g(x)$ in the sense of SSD. To complete the picture, Theorem 2 in Hadar and Russell (1971) shows that individuals with risk-averse preferences will prefer $f(x)$ to $g(x)$.[21] For our application, this implies that individuals will prefer the distribution of MPCRs, and hence contribute more, under the Baseline. 


\section{NOTES}

1. The public goods environment of a common pool resource game also includes elements of added uncertainty. Ostrom et al. (1992) study the 'social dilemma' of the common pool resource game in an experimental setting, while Walker and Gardner (1992) introduce probabilistic destruction of the resource into their experiments. The probability of destruction of the common pool resource in Walker and Gardner is an increasing function of total group exploitation of the resource, and such destruction ends the experiment. Walker and Gardner are quite similar to the VCM 'Incentive' treatment introduced in this paper in that the probability of a zero public good payoff is a function of aggregate contribution levels. The main difference is that in the Incentive treatment of this paper the probability of a zero public good payoff decreases in aggregate contributions. Furthermore, an instance of nonprovision of the public good in this paper does not end the experiment (since many group interaction environments realistically continue even after 'bad' outcomes).

2. Other authors have modeled different types of uncertainty in the VCM and show that risk aversion implies an increase in contributions. Austen-Smith (1980) looks at the uncertainty of the contributions of others, and Eichberger and Kelsey (1996) look at one shot games where it is difficult to assign precise probabilities to others' behavior. These represent different types of uncertainty from what is considered here.

3. Keep in mind that the payoffs here are not always money amounts. Payoffs in the first and second examples reflect both monetary payoffs and nonmonetary payoffs of being a member of a successful group effort. In the third example, the benefits may be almost entirely nonmonetary.

4. While winning a championship is a public good of a fixed size, there are different levels of achievement that are smaller sized public goods leading up to the championship (e.g. in professional baseball teams first win a division championship, then a league championship, and then the World Series). One could also consider the public good as being a team of a given quality. As increased effort is expended by team members, there will be a higher probability of receiving a larger public good (in terms of quality of play).

5. For a survey of treatment variables that have been shown to affect contribution levels in the VCM environment, see Ledyard (1995).

6. While neutral language is primarily used in presenting this environment to subjects in experimental instructions, the term 'contributions' does appear in the instructions in reference to total tokens in the public account. Some researchers now avoid these terms. In this experiment, consistency in the language across experimental treatments still allows a valid comparison of the treatment effects. Instructions are available upon request.

7. Restricting subjects' preferences to concave quadratic utility functions would allow us to predict the behavior of the subjects by simple-variance analysis. However, it is well known that there exists concave nonquadratic utility functions (i.e. still risk-averse individuals) such that those individuals would prefer a gamble of higher variance. In this case I show in A that less restrictive assumptions can be placed on individuals' preferences so that all subjects would prefer the Baseline, in terms of higher contribution 
rates, to the Uncertainty design in the sense of second-degree stochastic dominance. See Hadar, J. and Russell, W., 1971. , Stochastic dominance and diversification. Journal of Economic Theory 3, pp. 288-305 Article | PDF (1038 K) | View Record in Scopus | Cited By in Scopus (31)Hadar and Russell (1971) for formal definitions and proofs of the properties of stochastic dominance.

8. The dominant strategy for risk-neutral individuals is to contribute zero when $E M<1$. In the Incentive treatment, however, the EM has been radically changed to

$$
\begin{aligned}
& E M=\frac{a}{q N}\left(2 \alpha \sum_{i} m_{i} / N w\right)_{\text {with }} \\
& \partial E M / \partial\left(\sum_{i} m_{i}\right)=a / q N(2 \alpha / N w)>0
\end{aligned}
$$

when $\alpha>0$ so that EM increases in the total number of contributions. With the parameterization used, EM is an element of the $[0,1.00]$ range which shows that the dominant strategy, in terms of expected payoff, is preserved at all but on point (where it is still a weakly dominant strategy when the last group token is contributed to the public good).

9. Since this design and its predictions assume risk neutrality, it is interesting to note that in the case of a group of strongly risk-averse people, the expected utility of the total group payoff could be maximized when all subjects contribute nothing to the group account. In other words, for a group of strongly risk-averse subjects, the proper criterion for valuation of payoffs may not be just the expected value independent of the variance of different possible outcomes.

10. All Instructions, decision sheets, and data are available from the author upon request.

11. The behavioral assumption in using the fixed effects model is that each separate experimental group represents nothing more than a parametric shift in the regression equation. The fixed effect will capture any unknown effects and also reflect the nonzero starting point that seems apparent in all voluntary contributions mechanism settings.

12. There is one group per experiment, each group makes 6 decisions per treatment ( 7 actually, but as is apparent in Eq. 7, the lagged variables mean that only the last 6 rounds in each treatment are used), 3 treatments per experiments, and 10 experiments for a total of (6)(3)(10)=180 observations.

13. Subjects were not told the number of rounds in each treatment, but it was probably fairly obvious since their decision sheets indicated space for 7 rounds per treatment.

14. Unfortunately, the coefficients on Uncert and Nopublic are sensitive to model specification. When Eq. 6 is estimated with an interaction variable that multiplies $\mathrm{EM}^{*}$ Incent (replacing the two variables separately), the coefficient on Nopublic is negative and significant, and the fixed effects are larger. All other parameter values are robust with respect to model specification.

15. Other experiments that confirm the direction of the MPCR effect are Kim and Walker (1984), Brown-Kruse and Hummels (1992), and Saijo and Tatamitani (1991).

16. The importance of such deviations from the group mean has been formally recognized before this study. A model of noncooperative provision of public goods is developed in Falklinger (1994) in which own-contributions relative to the contributions of others enters into the payoff function. Additionally, Falklinger et al. (1995) experimentally examine a 
mechanism whereby deviations from the mean level of contributions are taxed and subsidized. Such a mechanism significantly increases contribution levels, but would not be viable in situations in which different endowments existed. Chan et al. (1996) also suggest a model that might lead to a similar variable. The difference with their model is that deviations are explicitly defined in terms of relative-to-endowment contributions.

17. There are 5 persons per experiment, each person makes 6 decisions per treatment $(7$ actually, but the first round in each treatment is thrown away), there are 3 treatments per experiment, and 10 experiments for a total of (3)(6)(5)(10)=900 observations.

18. The autocorrelation present in the group data was not present in the individual data. The estimated correlation coefficient for the individual level model was -0.01 .

19. This explanation was suggested to me by Stan Reynolds. Another more colorful possibility is that below-average contributors will free-ride even more while aboveaverage contributors will 'stoke the fire' by contributing more in continued efforts to raise others' contributions.

20. Since Eq. 7 imposes the same parameter on a positive deviation from the average as a negative one, an additional model was estimated in which a variable Interact is included. Interact is merely Deviate multiplied by a dummy variable that equals one if Deviate is positive. This allows us to find the effect on contributions of being an above-average contributor by summing the coefficients of Interact and Deviate. Results here still show that the parameter estimate on a positive deviation from the group average is still positive.

21. Specifically, it holds for preferences that are bounded, strictly increasing, possessing continuous derivatives of order one and two at each point in $R$ with the first derivative being finite, and the second derivative being nonpositive everywhere in $R$. This is less restrictive that the set of all concave and quadratic preferences. 